\title{
Anatomical variation of the extracranial course of the optic nerve in the floor of the sphenoid sinus: first reported case
}

\author{
A GIRGUIS-BUCHER, C SCHLEGEL-WAGNER
}

Department of Otolaryngology, Head and Neck Surgery, Lucerne Cantonal Hospital, Switzerland

\begin{abstract}
Objective: We report a unique case of anatomical variation of the extracranial course of the optic nerve running in the floor of the sphenoid sinus.

Method: Clinical and radiological findings are presented.

Results: A 39-year-old woman with Turner syndrome presented with severe headache associated with visual disturbances. Magnetic resonance imaging revealed a mass presumed to be a sella meningioma. Computed tomography of the paranasal sinuses was undertaken to help plan surgical removal via an endoscopic trans-sphenoidal approach; this scan revealed an atypical extracranial course of the optic nerve, running in the floor of the sphenoid sinus.
\end{abstract}

Key words: Optic Nerve; Paranasal Sinuses; Endoscopic Surgical Procedures; Tomography, X-ray Computed

\section{Introduction}

The optic nerve has an intimate relationship to the posterior ethmoid cells and sphenoid sinus. Protrusion of the optic

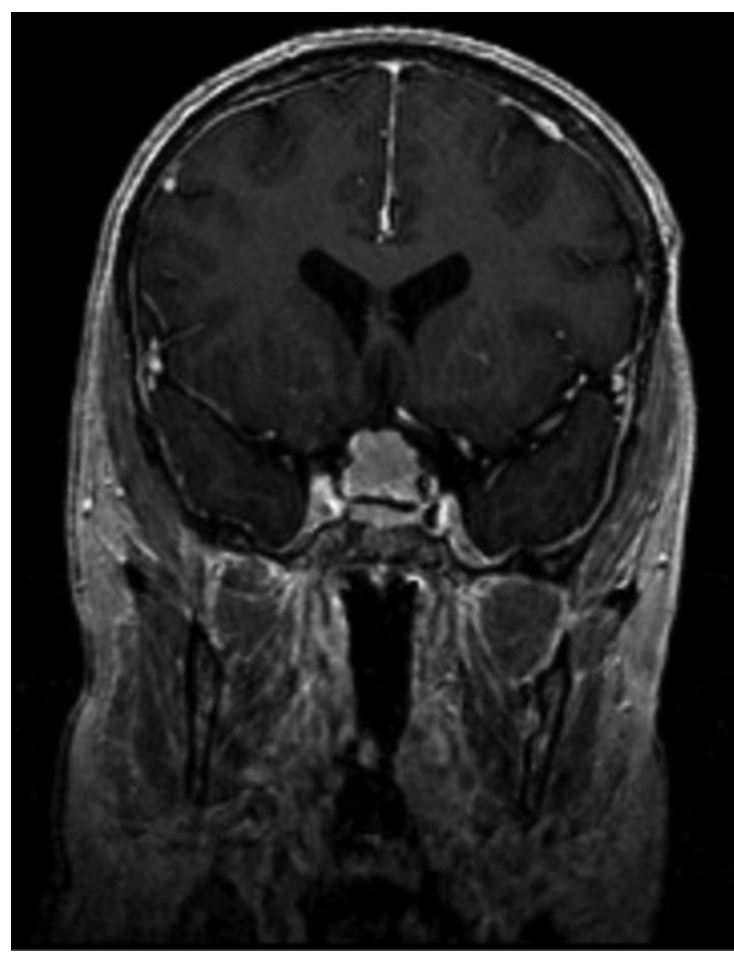

FIG. 1

Coronal, T1-weighted magnetic resonance imaging head scan with gadolinium contrast, showing the hyperintense lesion. canal into the sphenoid and/or ethmoid sinus (e.g. in an Onodi cell, or due to an atypical extracranial course of the optic nerve) has considerable clinical significance. Damage of the optic nerve is one of the most serious complications of endoscopic sinus surgery.

It is essential that clinicians performing sinus surgery have a detailed knowledge of the paranasal sinuses and also of the adjacent anatomical structures and their known variations. It is important to review such knowledge pre-operatively, so that unintentional damage to these structures can be avoided.

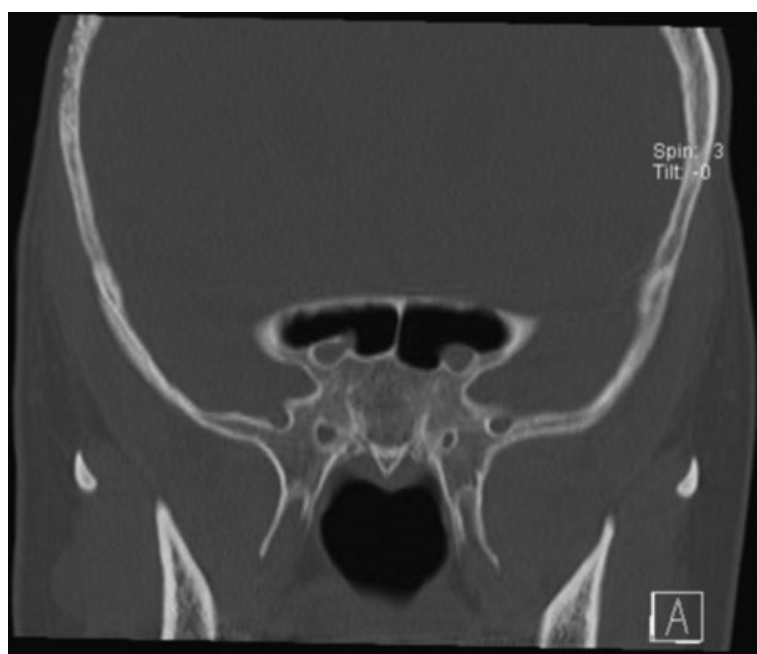

FIG. 2

Coronal computed tomography scan of the paranasal sinuses, showing a previously unreported anatomical variation of the extracranial course of the optic nerve, riding on the floor of the sphenoid sinus. 


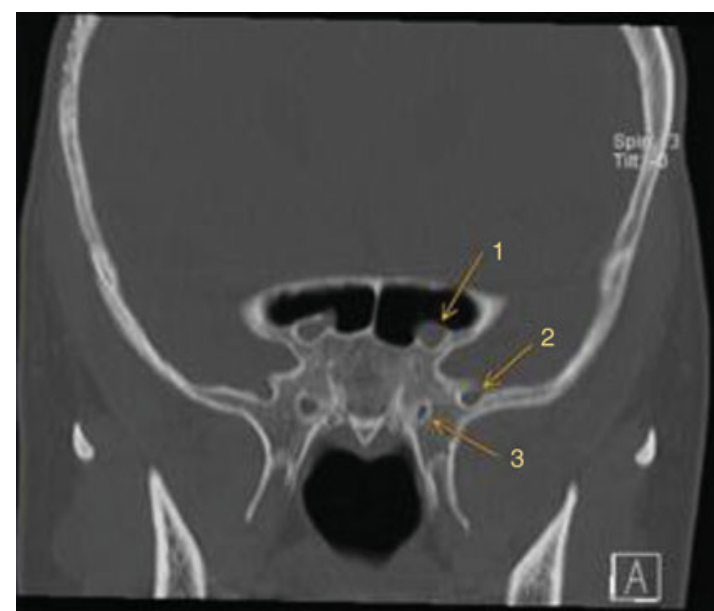

FIG. 3

Coronal computed tomography scan showing anatomical variation of the optic nerve course. $1=$ optic nerve; $2=$ maxillary nerve; $3=$ vidian nerve

\section{Case report}

A 39-year-old woman with Turner syndrome presented with a recent episode of severe headache associated with visual disturbances.

Ophthalmological examination revealed papilloedema in both eyes, with no other pathology. Other than Turner syndrome, the patient's medical history was unremarkable.

Magnetic resonance imaging of the brain revealed a large $(19 \times 6 \mathrm{~mm})$, well circumscribed mass in the region of the optic chiasm on the diaphragma sellae. The lesion was hyperintense on T1-weighted images with gadolinium contrast (Figure 1) and isointense on T2-weighted images. The mass was presumed to be a meningioma.

A computed tomography (CT) scan of the paranasal sinuses was undertaken to help plan surgical removal via an endoscopic trans-sphenoidal approach (Figure 2). Surprisingly, it revealed a previously unreported anatomical variation of the extracranial course of the optic nerve, riding on the floor of the sphenoid sinus.

Surgery was uneventful.

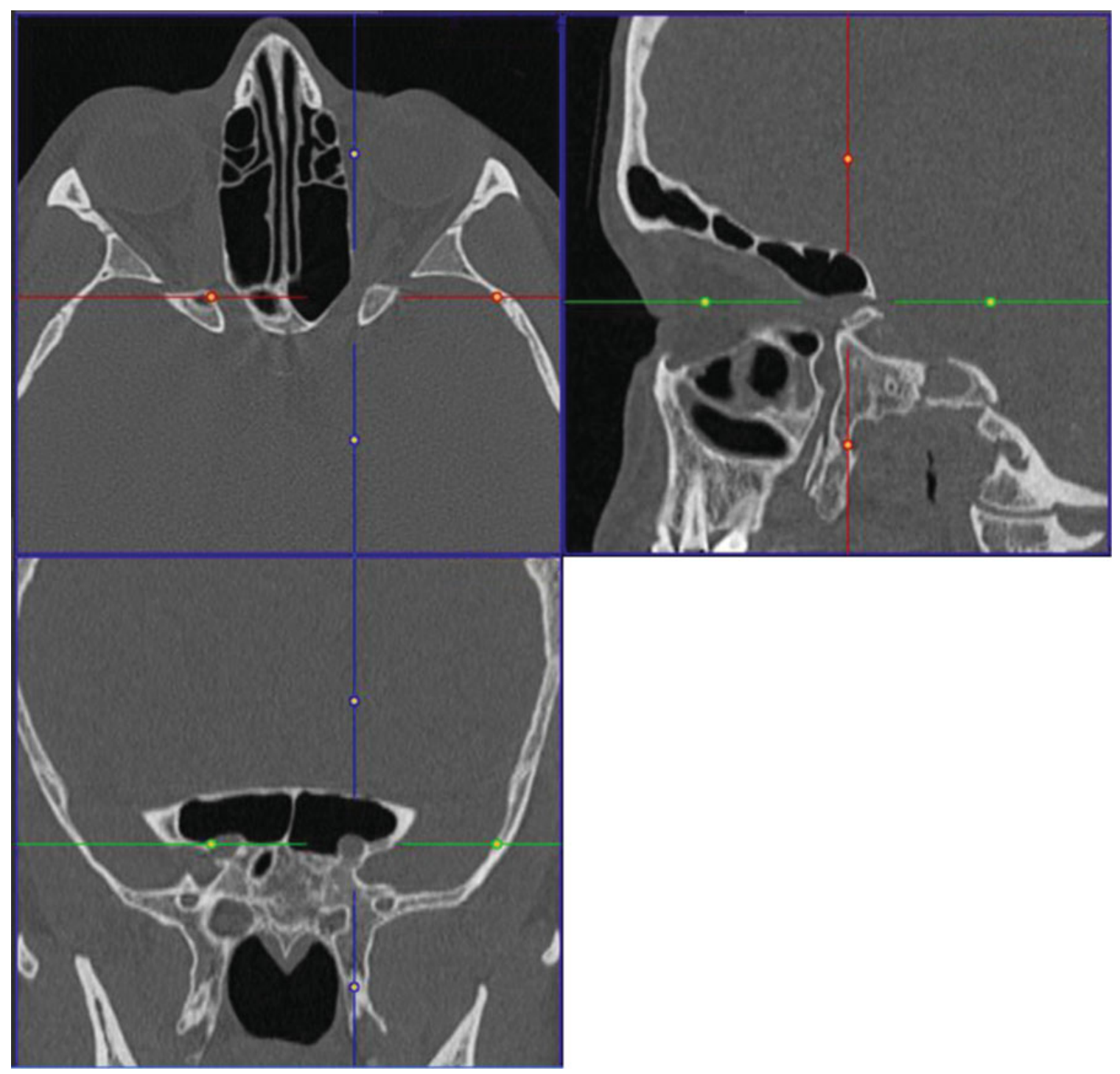

FIG. 4

Composite presentation of axial, sagittal and coronal computed tomography scans centred on the optic nerve, showing its variant course in three dimensions. 
Histopathological examination confirmed that the lesion was a sella meningioma.

\section{Discussion}

Turner syndrome is a common chromosomal abnormality characterised by complete or partial X chromosome monosomy. Schoemaker et al. ${ }^{1}$ performed a cohort study of Turner syndrome patients, and observed increased prevalence of various neoplastic disorders including meningioma, childhood brain tumour and gonadoblastoma. Interestingly, these patients showed a decreased risk of breast cancer. Such risks are postulated to relate either to direct genetic factors or to hormonal therapies used to treat the condition.

- Optic nerve damage is one of the worst complications of endoscopic sinus surgery

- In the presented case, the optic nerve coursed along the floor of the sphenoid sinus

- This anatomical variation has not been previously reported

- Pre-operative computed tomography is mandatory prior to endoscopic sinus surgery, to detect anatomical variation

Patients undergoing endoscopic sinus surgery must receive pre-operative $\mathrm{CT}$ scanning, particularly when a trans-sphenoidal approach is anticipated. It is usually advised that these pre-operative CT scans be evaluated using a checklist. ${ }^{2}$ Such CT scans provide a wealth of information on the patient's bony anatomy, giving surgeons a detailed map with which to plan their approach. Knowledge of anatomical variations may be crucial. In particular, knowledge of the anatomical course of the optic nerve is of great importance in order to avoid injury while performing an endoscopic trans-sphenoidal approach. The usual anatomical course of the optic nerve is within the supero-lateral aspect of the wall of the sphenoid sinus.
The presented patient's variant anatomy prompted us to search the literature, using the PubMed database, to identify previous reports of anatomical variation of the extracranial course of the optic nerve. Variant courses have been previously reported, including cases in which the optic nerve bulged into the sinus or even traversed it. ${ }^{3-5}$ However, we could find no previous report of the optic nerve occupying such a low position within the floor of the sinus. Figures 3 and 4 show further detail of this previously unreported anatomical variation.

\section{References}

1 Schoemaker MJ, Swerdlow AJ, Higgins CD, Wright AF, Jacobs PA, UK Clinical Cytogenetics Group. Cancer incidence in women with Turner syndrome in Great Britain: a national cohort study. Lancet Oncol 2008;9:239-46

2 Simmen D, Schuknecht B. Computerized tomography of paranasal sinuses - a preoperative checklist [in German]. Laryngorhinootologie 1997;76:8-13

3 Heskova G, Mellova Y, Holomanova A, Vybohova D, Kunertova $\mathrm{L}$, Marcekova $\mathrm{M}$ et al. Assessment of the relation of the optic nerve to the posterior ethmoid and sphenoid sinuses by computed tomography. Biomed Pap Med Fac Univ Palacky Olomouc Czech Repub 2009;153:149-52

4 De Lano MC, Fun FY, Zinreich SJ. Relationship of the optic nerve to the posterior paranasal sinuses: a CT anatomic study. Am J Neuroradiol 1996;17:669-75

5 Dessi P, Moulin G, Chagnaud C, Cannoni M. Protrusion of the optic nerve into the ethmoid and sphenoid sinus: prospective study of 150 CT studies. Neuroradiology 1994;36:515-16

Address for correspondence:

Dr. Andrea Girguis Bucher,

HNO medic,

Splügenstrasse 6 ,

8002 Zurich, Switzerland

Fax: +41442010056

E-mail: girguis@hno-medic.ch

Dr A Girguis-Bucher takes responsibility for the integrity of the content of the paper

Competing interests: None declared 